\title{
Strain Specific: Microbial Strains Involved in Gut-Brain Signaling
}

Jessica N. Keating ${ }^{1}$, Brittney Patterson ${ }^{1}$, Roberta Speir ${ }^{1}$, Caroline Wiswell ${ }^{1}$, Luz Aceves Gonzalez ${ }^{1}$, Hema Kundargi ${ }^{1}$, Tugba Bayrak ${ }^{1}$, Colette LaSalle ${ }^{1}$

Exploration into the microbial role within behavior and neurologic regulation has been an area of growing interest and research. While in-vitro and in-vivo experimentation has suggested that commensal microbiota play a role within behavioral and neurologic functioning, little distinction has been made about the specific microbes inducing change. In order to understand and potentially utilize this complex gut-microbe-brain connection, it is imperative to distinguish between which microbes are inducing behavioral and or neurologic effects, and which biologic mechanisms are mediating said effects. To enhance the current understanding of neurologically influential microbes, this review will analyze eight microbial strains belonging to the genus types Lactobacillus and Bifidobacterium, and note the similarities and dissimilarities pertaining to modulation of inflammatory response, intestinal permeability, neurochemical concentrations, and interaction with the vagus nerve expressed amongst included microbial strains. Analysis of the selected microbes demonstrated significant distinction regarding neurochemical, inflammatory, and immunological effect amongst microbial strains belonging to the genus types Lactobacillus and Bifidobacterium. Interestingly, despite the expressed biologic variation, behavioral influence was largely uniform amongst the included microbial strains and expressed almost exclusively through a reduction in typified anxious and depressive behavior.

\section{INTRODUCTION}

Despite the myriad of pills and procedures aiming to treat psychiatric abnormalities and conditions, there is still much to be discovered about the brain. Fortunately, exploration into the effect upon commensal microbiota within behavioral and neurologic regulation has created a path in which to further decode and explore this enigmatic terrain. Recent in vitro and in vivo experimentation has demonstrated microbial influence within complex emotive states such as depression, chronic stress, anxiety, and psychiatric disorder (Bailey et al., 2011; Bercik et al., 2010; Maes, Kubera, Leunis, \& Berk, 2012; Rook \& Lowry, 2008). While this field is emerging and many mechanistic factors facilitating the microbial influence within gut-brain axis regulation have yet to be identified, the work done thus far suggest a future in which the brain can be indirectly targeted for therapeutic benefit through manipulation of commensal microbiota (Clarke et al., 2014; Cryan \& Dinan, 2012). At a time when neuropsychiatric disorders are the leading cause of dis-

${ }^{1}$ San Jose State University, 1 Washington Square, San Jose, California, 95192

*To whom correspondence should be addressed: jesskeating@comcast.net

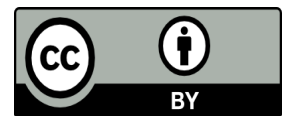

Except where otherwise noted, this work is licensed under https://creativecommons.org/licenses/by/4.0/

doi:10.22186/jyi.33.3.49-54 ability in the U.S (USBDC, 2013), the importance of this research cannot be overstated.

Although the blueprint outlining the microbial role within gut-brain axis regulation is far from maturation, the current understanding is that certain microbial strains are able to induce measurable neurologic and behavioral effect through the modulation of inflammatory response, neurochemical concentration, barrier-integrity, and interaction with the vagus nerve. In order to understand, and potentially utilize these microbial capabilities, it is essential to distinguish between which microbes are inducing behavioral and or neurologic effect, and which pathway each microbe is using to do so. The complexity of this task resides in the significant microbial distinction expressed not only on a species level, but amongst microbial strains (Greenblum, Rogan, \& Borenstein, 2015).

Among the more than 7,000 microbial strains (Ley, Peterson, \& Gordon, 2006), majority have not demonstrated direct behavioral or neurologic effect. The collection of strains that have been shown to induce measurable neurologic and behavioral manipulation belong to one of the three genus types Lactobacillus, Bifidobacterium, and Bacteroides (Mayer, Knight, Mazmanian, Cryan, \& Tillisch, 2014). Amongst these genus types, a plethora of biologic and behavioral variances have been expressed on both a species and strain level. While variation across differing genus and species type is expected, differences expressed between microbial strains belonging to the same genus and species type is a surprising find in light of the genomic similarity expressed between them. Further investigation into these microbial variances can explain questions such as why, despite genomic similarities, only certain microbial strains are able to induce behavioral and neurologic effect. Why 
the microbial strains that do induce measurable effect, do so in a variety of ways that are often dissimilar from other strains belonging to the same genus and species type. And whether or not these strain mediated effects can be utilized for therapeutic benefit within neurologic and psychiatric disorders.

\section{Lactobacillus rhamnosus JB-1.}

L. rhamnosus JB-1 induces effect within gut-brain signaling through modulation of GABA receptors and inflammatory response. In an experimental study by Bravo et al. (2001), chronic treatment of L. rhamnosus JB-1 in a healthy mice model significantly increased GABA-B receptor concentrations in the cingulate and pre-limbic cortical regions, increased the concentration of GABA-A receptors in the hippocampus, decreased GABA-B receptor concentrations in the hippocampus, amygdala, and locus coerulus, and decreased GABA-A receptors concentrations in the pre-frontal cortex and amygdala. The influence over GABA receptors is supported to have psychiatric impact that is demonstrated through work by Cryan and Kaupmann (2005), whom found a reduced concentration of GABA-B receptors in the frontal cortices of a depression induced mouse model, and Jacobson-Pick, Elkobi, Vander, Rosenblum, and Richter-Levin (2008) whom found an increase of GABA-A receptors in the amygdala of a chronically stressed mice model.

Inflammatory affect was demonstrated in a study by Forsythe and Bienstock (2004), which found that L. rhamnosus JB-1 reduced intestinal inflammation through an upregulation of nerve growth factor, and inhibition of IL-8 synthesis. The neurologic impact of these noted effects is supported in work done by Angelucci, Aloe, Vasquez, and Mathé (2000), showing an altered concentration of brain derived neurotropic factor and nerve growth factor in a depression induced mice model. As well as in work done by Janelidze et al. (2015), showing an association between low serum levels of IL-8 and anxiety in suicidal patients.

L. rhamnosus JB-1 demonstrated behavioral impact in the abovementioned study by Bravo et al. (2001) through a significant decrease in typified anxious and depressive behavior in mice chronically administered with $L$. rhamnosus JB-1. In addition to modulation of GABA receptors, this effect was deemed to be in relation to interaction with the vagus nerve, for behavioral effect was not observed in vagotamized mice (Bravo et al., 2001). Due to the noted associating between nerve growth factor and depression, the increased concentration of nerve growth factor by L. rhamnosus JB- 1 could also play a role within the noted decrease of typified anxious and depressive behavior.

\section{Lactobacillus rhamnosus GG.}

L. rhamnosus GG induced neurologic influence primarily through modulation of inflammatory processes. In an experimental study by Turner (2009), children with atopic dermatitis administered with L. rhamnosus GG resulted in significant reductions of serum levels of IL-10, which has been shown to reduce intestinal inflammation through the suppression of regulatory T cells (Park et al., 2005). The behavioral impact of this effect is suggested in a previous study by Dhabhar et al. (2009), which observed a decreased concentration of IL-10 levels in adults with major depression syndrome. Additional inflammatory effect was observed in an experimental study by Pessi et al. (2001), which noted a decreased concentration of regulatory $\mathrm{T}$ cells in casein degraded by L. rhamnosus GG. While no direct associations between regulatory $\mathrm{T}$ cell concentration and behavioral and or neurologic impact was observed, an indirect link pertaining to decreased inflammation by regulatory T cells (Park et al., 2005) which has been correlated with depression and anxiety (Bercik et al., 2010; Maes, Kubera, Leunis, \& Berk, 1999; Smith \& Rudolph, 1991;) supports the psychiatric influence of this inflammatory effect.

Behavioral impact was observed in a study by Kantak, Bobrow, and Nyby (2013), which observed an attenuation of obsessive compulsive disorder (OCD) typified behaviors in a house mice model administered with $L$. rhamnosus $\mathrm{GG}$, that worked as well as the positive study control, fluoxetine, which is common medication used in OCD treatment (Bandelow et al., 2012). The noted behavioral affect was presumed to be in relation to a decrease in the anxiety often preceding or accompanying OCD, and not from a direct modulation of the neurologic mediators associated with OCD typified behavior (Kantak et al., 2013). In reference to the abovementioned effects upon inflammation, it is plausible to infer that the noted behavioral modulation could be mediated, at least partially, through inflammatory modulation which has been previously associated with anxiety (Maes et al., 1999; Smith \& Rudolph, 1991).

\section{Lactobacillus helveticus NS8.}

L. helveticus NS8 induces behavioral and neurologic impact through modulation of neurotransmitters and inflammatory response. Research conducted by Liang et al. (2015) found that when administered in a pathogen free rat model, L. helveticus NS8 restored hippocampal concentrations of serotonin and norepinephrine. The psychological significance of this effect can be noted in previous work by Stanton and Sarvey (1985), which found that hippocampal depletion of norepinephrine reduces the frequency and magnitude of long term potentiation, which is a synaptic mechanism associated with cognitive functions pertaining to learning and memory (Eichenbaum \& Otto, 1992), as well as attention and arousal (Shors \& Matzel, 1997). Hippocampal effect was also noted in a study by Martinowich et al (2007), which observed an increase of hippocampal brain derived neurotrophic factor mRNA, which has been noted to play a role within depression and psychiatric disorders such as schizophrenia and bi-polar disorder (Martinowich et al., 2007; Palomino et al., 2006). L. helveticus NS8 was also shown to induce inflammatory effect through the attenuation of LPS induced inflammation which was mediated through an increased synthesis of IL-10 (Rong et al., 2015).

L. helveticus NS8 induced behavioral effect in an experimental study by Luo et al. (2014), which observed an attenuation of anxiety and improved cognition in a hyperammonemia induced rat model administered with $L$. helveticus NS8. These results were supported in a proceeding study by Liang et al. (2015), which ob- 
served a significant reduction of chronic stress induced anxiety, depression, and cognitive dysfunction in mice orally administered with $L$. helveticus NS8. All abovementioned induced effects including the synthesis of serotonin and norepinephrine (Liang et al., 2015), increased concentration of hippocampal brain derived neurotrophic factor mRNA (Martinowich, Manji, and Lu, 2007), and increased synthesis of IL-10 (Rong et al., 2015), appear to be plausible, and likely, mechanisms mediating the observed behavioral impact by L. helveticus NS8.

\section{Lactobacillus helveticus R0052.}

L. helveticus R0052 induced cerebral effect through mechanisms pertaining to increased barrier integrity and amelioration of stress induced irregularities in hypothalamic-pituitary-adrenal axis (HPA-axis) and automatic nervous system (ANS) functioning. Influence upon barrier integrity was supported in a study by Johnson-Henry, Hagen, Gordonpour, Tompkins, and Sherman (2007), which observed that $L$. helveticus $\mathrm{R} 0052$ prevented against pathogenic infiltration of Escherichia coli $0157: \mathrm{H} 7$ by binding to epithelial cells and blocking pathogenic entry. This impact suggests that $L$. helveticus R0052 may play a beneficial immunologic role through the enhancement of intestinal barrier security and protection against pathogenic infection. This is a neurologically relevant action, for previous studies have shown a connection between pathogenic infection and behavior (Quinn et al., 1984) as well as psychological stress and barrier integrity (Ait-Belgnaoui, Bradesi, Fioramonti, Theodorou, \& Bueno, 2005). HPA-axis modulation was supported in a study by (Ait-Belgnaoui et al., 2014), which observed a reversal of stress-induced HPA-axis and ANS irregularities, which was mediated through a decreased plasmatic concentration of corticosterone, adrenaline, and noradrenaline in a stress-induced mice model administered with a combination of $L$. helveticus R0052 and Bifidobacterium longum R0175. The modulation of the HPA-axis presents possible psychological relevance, for the HPA-axis has domain over coordinating all bodily stress responses (Tsigos \& Chrousos, 2002).

L. helveticus R0052 induced behavioral effect in a study by Ohland et al. (2013), which observed an amelioration of diet-induced anxiety and memory deficits in mice administered with $L$. helveticus R0052. Interestingly, this effect appeared to be diet-dependent, for behavioral effect was only noted when mice were followed a high fat "western" diet, but not when following a low fat "chow" diet. These results were supported in another study by Gilbert, Arseneault-Bréard, and Flores Monaco (2013), which noted a diet-dependent decrease in typified depressive behavior in a post-myocardial infarction induced mice model administered with a combination of $L$. helveticus R0052 and Bifidobacterium longum R0175. Although future experimentation is required to be able to accurately ascertain the likely factors mediating the noted behavioral influence, it is reasonable to conclude that in addition to dietary intake, the abovementioned influence over HPA-axis regulation (Ait-Belgnaoui et al., 2014) could be a contributing factor to the noted behavioral modulation.

\section{Lactobacillus johnsonii N6.2}

L. johnsonii N6.2 induced neurologic effect through modulation of neurotransmitters concentrations, gut-barrier integrity, and oxidative stress concentrations. In an experimental study by Valladares et al. (2013), L. johnsonii N6.2 increased ileum and peripheral serotonin concentrations, decreased peripheral kynurenine concentrations, and decreased tryptophan activity when administered to a "BioBreeding" rat model. The significance of this alteration within behavior and neurologic functioning can be seen in previous work showing an association between low levels of serotonin and depression (Lucki et al., 1998), as well as an association between tryptophan depletion and depression and panic disorder symptom relapse (Bell et al., 2001). Further microbial influence was shown in a study by Valladares et al. (2010), which observed that when administered to a diabetic induced rat model, L. johnsonii N6.2 induced effect within barrier integrity by increasing concentrations of tight junction protein claudin and decreasing host oxidative stress response. In addition to the previously noted association between barrier integrity and psychological stress (Ait-Belgnaoui et al., 2005), the behavioral significance of the modulation of oxidative stress response is supported in a study by Gorrindo, Lane, Lee, McLaughlin, and Levitt (2013), which found a correlation between increased oxidative stress response levels and increased severity of autism spectrum disorder associated behavioral abnormalities pertaining to language impairment, OCD typified behavior, and aversion to social communication. While no studies have tested to see whether or not L. johnsonii N6.2 induces effect within behavior, the noted induced effects pertaining to modulation of neurotransmitters and effect upon oxidative stress levels warrants future research to assess the degree of possible behavioral modulation.

\section{Bifidobacterium longum, subspecies longum JCM1217.}

Bifidobacterium longum subsp. longum JCM1217 induced effect within gut-brain axis regulation through immunological modulation. In a study by Fukuda et al. (2011), pathogenic infection of Escherichia coli 0157:H7 was prevented in mice administered with $B$. longum, subspecies longum JCM1217 through production of acetate. Fukuda et al. (2011) proposed that increased acetate production protected against pathogen infection by attaching to epithelial cells and preventing the pathogen from translocating from the gut lumen to host blood supply. In addition to the increased barrier integrity, which has been previously noted to have an association with psychological stress (Ait-Belgnaoui et al., 2005), the induced production of acetate could have possible behavioral ramifications, for experimentation by MacFabe et al. (2007) observed that autism spectrum disorder behavioral abnormalities were induced in a rat model when short chain fatty acid levels were manually increased. More research should be conducted on B. longum, subspecies longum JCM1217 to assess whether or not the noted immunological effect induces behavioral or neurologic modulation. 


\section{Bifidobacterium longum NCC3001.}

In an experimental study by Bercik et al. (2010), Bifidobacterium longum NCC3001 ameliorated colitis associated behavioral alterations and brain derived neurotrophic factor depletions, which has been previously associated with stress and depression (Martinowich et al., 2007; Angelucci et al., 2000). However, when tested in a follow-up study by Bercik et al. (2011), colitis associated alterations brain derived neurotrophic factor was not observed, which demonstrates that this was likely not a factor mediating the noted behavioral change. Additional neurologic effect was demonstrated in a study Khoshdel et al. (2013), which observed that B. longum NCC3001 significantly reduced the excitability of enteric neurons when applied to ileal segments of adult mice. This effect upon enteric neurons was supported in a study by Bercik et al. (2011), and presumed to be a possible method in which the central nervous system is signaled through activation of vagal pathways within the enteric nervous system.

Behavioral effect was noted in the abovementioned studies (Bercik et al., 2010; Bercik et al., 2011) through the attenuation of colitis associated typified anxious behavior. Both studies also observed in the abovementioned study by Bercik et al. (2011) through an attenuation of typified anxious behavior and an increase in typified exploratory behavior. Interaction with the vagus nerve appeared unclear, for behavioral effect was found independent of the vagus nerve in the first study by Bercik et al. (2010) and dependent of the vagus nerve in the follow-up study by Bercik et al. (2011). While the noted effect regarding decreased excitability of the vagus nerve supports interaction with the vagus nerve within behavioral modulation (Bercik et al., 2010; Khoshdel et al., 2013), more research is required to adequately determine the degree of vagal involvement.

\section{Bifidobacterium infantis 35624.}

Bifidobacterium infantis 35624 induced both behavioral and neurologic effect through modulation of neurotransmitters, inflammatory response, and intestinal permeability. Research by Desbonnet, Garrett, Clarke, Bienenstock, and Dinan (2008) observed that $B$. infantis 35624 reduced frontal cortex serotonin concentrations, increased plasmatic concentrations of noradrenaline and tryptophan, and inhibited the production of IL-10. The significance of these mediated effects are supported in previous work by (Amat et al., 2005; Bland et al., 2003) showing a connection between prefrontal cortex serotonin concentration and the modulation of anxiety, in work by Vijayakumar and Meti (1999) showing a connection between noradrenaline and depression, in work by Myint et al. (2007) showing a connection between decreased plasmatic tryptophan concentrations and depression, and work by (Dhabhar et al., 2009) showing a connection between IL-10 and major depressive syndrome.

Behavioral influence was demonstrated in the abovementioned study by (Desbonnet et al., 2008) which noted an amelioration of typified depressive behavior in rats administered with $B$. infantis 35624 that worked as effectively as the positive study control Citalopram supporting the effectiveness of B. infantis 35624 as a behavioral regulator.

\section{DISCUSSION}

The variation of induced effects expressed by the 8 included microbial strains demonstrate that even amongst strains belonging to the same genus type, generalizations pertaining to induced effects should be avoided. For example, while four out of the five included Lactobacillus strains induced influence over a collection of 9 neurotransmitters, modulation over the same neurotransmitter was only expressed one time in the modulation of serotonin by both L. helveticus NS8 and L. johnsonii N6.2 (Liang et al., 2015; Valladares et al., 2013). Interestingly, in some instances microbial strains belonging to the same genus type induced oppositional effects. For example, while both L. rhamnosus GG and L. helveticus NS8 impacted the concentration of anti-inflammatory cytokine IL-10, L. rhamnosus GG decreased IL-10 concentrations (Turner., 2009) while L. helveticus NS8 increased them (Rong et al., 2015). Oppositional impact was also expressed amongst strains belonging to differing genus types, such as L. johnsonii N6.2, which increased peripheral serotonin concentrations (Valladares et al., 2013), and B. infantis, which reduced peripheral serotonin concentrations (Desbonnet et al., 2008).

Despite the diversity of microbially induced effects, behavioral modulation was largely uniform amongst the included strains. Six out of the eight strains demonstrated behavioral influence through the attenuation of of typified anxious and or depressive behavior. The apparent dichotomy expressed by the divergent mediated effects and uniform behavioral influence demonstrates the complexity of assessing the microbe-brain connection. As with many organically occurring events, no one factor determines whether or not an effect will take place. It is a consortium of many factors, some yet to be identified, and some yet to be understood.

While the noted behavioral modulation is promising and undoubtedly warrants further investigation, it is essential to remember the vast differences between human and animal physiologies and the methodology in which behavioral influence is assessed in animal models. The clinical testing done thus far has suggested promising results. For example, a study examining the effect of a multispecies probiotic supplement upon depressive indicators in healthy individuals found a significant decrease in a cognitive reactivity to sad mood amongst those given the probiotic supplement when compared to participants supplied with the placebo (Steenbergen, Sellaro, Hemert, Bosch, \& Colzato, 2015). This effect was attributed to a decrease in frequency of negative and aggressive thoughts which further supports the potential therapeutic benefit of varying microbial strains (Steenbergen et al., 2015). It will be interesting to see whether or not this effect is viable in participants who have pre-existent conditions such as depression or acute anxiety as well as other psychiatric disorders.

\section{ACKNOWLEDGEMENTS}

This review was completed as an exterior project for a graduate level course facilitated by Dr. Colette LaSalle, at San Jose State University. 


\section{REFERENCES}

Ait-Belgnaoui, A., Bradesi, S., Fioramonti, J., Theodorou, V., \& Bueno, L. (2005). Acute stress-induced hypersensitivity to colonic distension depends upon increase in paracellular permeability: role of myosin light chain kinase. Pain, 113, 141-147. doi:10.1016/j.pain.2004.10.002

Ait-Belgnaoui, A., Colom, A., Braniste, V., Ramhalo, L., Marrot, A., Cartier, C., \& Tompkins, T. (2014). Probiotic gut effect prevents the chronic psychological stress-induced brain activity abnormality in mice. Neurogastroenterology \& Motility, 26, 510-520. doi:10.1111/nmo.12295

Amat, J., Baratta, M. V., Paul, E., Bland, S. T., Watkins, L. R., \& Maier, S. F. (2005). Medial prefrontal cortex determines how stressor controllability affects behavior and dorsal raphe nucleus. Nature Neuroscience, 8, 365-371. doi:10.1016/S0079-6123(03)46011-1

Angelucci, F., Aloe, L., Vasquez, P. J., \& Mathé, A. A. (2000). Mapping the differences in the brain concentration of brain-derived neurotrophic factor (BDNF) and nerve growth factor (NGF) in an animal model of depression. NeuroReport, 11(6): 1369-73. doi:10.1097/00001756-200004270-00044

Angelucci, F., Aloe, L., Vasquez, P. J., \& Mathé, A. A. (2000). Neurotrophic factors and CNS disorders: findings in rodent models of depression and schizophrenia. NeuroReport, 11(6), 1369-73. doi:10.1016/S0079-6123(03)46011-1

Backhed, F., Ding, H., Wang, T., Hooper L.V., Koh, G. Y., Nagy, A., Semenkovich, C. F., \& Gordon. J. I. (2004). The gut microbiota as an environmental factor that regulates fat storage. Proceedings of the National Academy of Sciences of the United States, 101, 15718-15723. doi:10.1073/pnas.0407076101

Bailey, M. T., Dowd, S. E., Galley, J. D., Hufnagle, A. R., Allen, R. G., \& Lyte, M. (2011). Exposure to a social stressor alters the structure of the intestinal microbiota: implications for stressor-induced immunomodulation. Brain Behavior Immunity, 25, 397-407. doi: 10.1016/j.bbi.2010.10.023

Bandelow, B., Sher, L., Bunevicious, R., Hollander, E., Kasper, S., Zohar, J., \& Moller, J. H. (2012). Guidelines for the pharmacological treatment of anxiety disorders, obsessive - compulsive disorder and posttraumatic stress disorder in primary care. International Journal of Psychiatry in Clinical Practice, 16, 77-84. doi:10.3109/13651501.2012.667114

Bell, C., Forshall, S., Adrover, M., Nash, J., Hood, S., Argyropoulos, S., Rich, A., \& Nutt, J. D. (2002). Does 5-HT restrain panic? A tryptophan depletion study in panic disorder patients recovered on paroxetine. Journal of Psychopharmacology 16(1), 5-14. doi:10.1177/026988110201600116

Bercik, P., Park, A. J., Sinclair, D., Khosdel, A., Huang, X., Deng, Y... \& Verdu, E. F. (2011). The anxiolytic effect of Bifidobacterium longum NCC3001 involves vagal pathways for gut-brain communication. Neurogastroenterology and Motility, 23,1132-9. doi:10.1111/j.1365-2982.2011.01796.x

Bercik, P., Verdu, E. F., Foster, J. A., Macri, J., Potter, M., Huang, X... \& Collins, S. M. (2010). Chronic gastrointestinal inflammation induces anxiety-like behavior and alters central nervous system biochemistry in mice. Gastroenterology, 139 (6), 2102-2112. doi:10.1053/j.gastro.2010.06.063

Bland, S. T., Hargrave, D., Pepin, J. L., Amat, J., Watkins, L. R., \& Maier, S. F. (2003). Stressor-controllability modulates stress-induced dopamine and serotonin efflux in the medial prefrontal cortex. Neuropsychopharmacology, 28, 1589-1596. doi:10.1038/sj.npp.1300206

Bravo, J. A., Forsythe, P., Chew, M. V., Escaravage, E., Savingnac, H. M., Dinan, T. G., Bienenstock, J. \& Cryan, J. F. (2001). Ingestion of Lactobacillus strain regulates emotional behavior and central GABA receptor expression in a mouse model via the vagus nerve. Proceedings of the National Academy of Sciences USA, 108, 16050-16055.

Clarke, G., Stilling, R. M., Kennedy, P. J., Stanton, C., Cryan, J. F., \& Dinan, T. G. (2014). Minireview: Gut Microbiota: The Neglected Endocrine Organ. Molecular Endocrinology, 28(8) 1221-1238. doi:10.1210/me.2014-1108

Cryan, J. F., \& Dinan, T. G., (2012). Mind-altering microorganisms: the impact of the gut microbiota on brain and behavior. Nature Reviews Neuroscience, 13(10), 701-712. doi:10.1038/nrn3346

Cryan, J. F., \& Kaupmann, K. (2005). Don’t Worry 'B' happy!: a role for GABA(B) receptors in anxiety and depression. Trends in Pharmaceutical Science, 26, 36-43.

Desbonnet, L., Garrett, L., Clarke, G., Bienenstock, J., \& Dinan, T. G. (2008). The probiotic Bifidobacteria infantis: an assessment of potential antidepressant properties in the rat. Journal of Psychiatric Research, 43,164-74. doi:10.1016/j.jpsychires.2008.03.009

Dhabhar, F. S., Burke, H. M., Epel, E. S., Mellon, S. H., Rosser, R., Reus, V. I., \& Wolkowitz, O. M. (2009). Low serum IL-10 concentrations and loss of regulatory association between IL-6 and IL-10 in adults with major depression. Journal of Psychiatric Research, 43(11), 962-9. doi:10.1016/j.jpsychires.2009.05.010

Eichenbaum, H., \& Otto, T. (1992). The hippocampus - What does it do? Behavioral and Neural Biology, 57, 2-36. doi:10.1016/0163-1047(92)90724-I

Forsythe, P., \& Bienstock, J. (2004). Live Lactobacillus rhamnosus [corrected] is essential for tumor factor necrosis factor alpha-induced interleukin-8 expression. Infection and Immunity, 72 (9), 5308-5314. doi:10.1128/IAI.72.9.53085314.2004

Fukuda, S. T., Toh, H., Hase, H., Oshima, K., Nakanishi, Y., Yoshimura, K., \& Ohno, H. (2011). Bifidobacteria can protect from enteropathogenic infection through production of acetate. Nature ,469 (7331), 543-547. doi:10.1038/ nature09646

Gilbert, K., Arseneault-Bréard, J., Flores., \& Monaco, F. (2013). Attenuation of post-myocardial infarction depression in rats by n-3 fatty acids or probiotics starting after the onset of reperfusion. British Journal of Nutrition, 109, 5056. doi: $10.1017 / \mathrm{S} 0007114512003807$

Gorrindo, P., Lane, C. J., Lee, E. B., McLaughlin, B. A., \& Levitt, P. (2013). Enrichment of Elevated Plasma F-Isoprostane Leves in Individuals with Autism Who Are Stratified by Presence of Gastrointestinal Dysfunction. PLOS one, 8(7) E6844. doi:10.1371/journal.pone.0068444

Greenblum, S., Rogan, C., \& Borenstein, E. (2015). Extensive Strain-Level Copy-Number Variation across Human Gut Microbiome Species. Cell, 160, 583-594.

Jacobson-Pick, S., Elkobi, A., Vander, S., Rosenblum, K., \& Richter-Levin, G. (2008). Juvenile Stress-induced Alteration of Maturation of the GABAA Receptor Alpha Subunit in the Rat. International Journal of Neuropsychopharmacology, 11(7):891-903. doi:10.1017/S1461145708008559

Janelidze, S., Suchankova, P., Ekman, A., Erhardt, S., Sellgren, C., Samuelsson, M., Westrin, A., Minthon, L., Hansson, O., Träskman-Bendz, L., \& Brundin, L. (2015). Low IL-8 is associated with anxiety in suicidal patients: genetic variation and decreased protein levels. Acta Psychiatrica Scand, 131(4), 26978. doi:10.1111/acps. 12339

Johnson-Henry, K. C., Hagen, K. E., Gordonpour, M., Tompkins, T. A., \& Sherman, P. M. (2007). Surface-layer protein extracts from Lactobacillus helveticus inhibit enterohaemorrhagic Escherichia coli O157:H7 adhesion to epithelial cells. Cell Microbiology, 9, 356-367.

Kantak, P. A., Bobrow, D. N., \& Nyby, J. G. (2013). Obsessive-compulsivelike behaviors in house mice are attenuated by a probiotic (Lactobacillus rhamnosus GG). Behavioural Pharmacology, 25(1), 71-79. doi:10.1097/ FBP. 0000000000000013

Khoshdel, A., Verdu, E. F., Kunze, W., McLean, P., Bergonzelli, G., \& Huizinga, J. D. (2013). Bifidobacterium longum NCC3001 inhibits AH neuron excitability. Neurogastroenterology and Motility, 7, e478-84. doi:10.1111/nmo.12147

Ley, R. E., Peterson, D. A., \& Gordon, J. I. (2006). Ecological and evolutionary forces shaping microbial diversity in the human intestine. Cell 124, 837-848. doi:10.1016/j.cell.2006.02.017

Liang, S., Wang, T., Hu, X., Luo, J., Li, W., Wu, X., Duan, Y., \& Jin, F. (2015). Administration of Lactobacillus Helviticus NS8 Improves Behavioral, Cognitive, and Biochemical Aberrations Caused by Chronic Restraint Stress. Neuroscience, 3(310), 561-5677. doi:10.1016/j.neuroscience.2015.09.033

Lucki I. (1998). The spectrum of behaviors influenced by serotonin. Biological Psychiatry, 44(3), 151-62. doi:10.1016/S0006-3223(98)00139-5

Luo, J., Wang, T., Liang, S., Hu, X., Li, W., \& Jin, F. (2014). Ingestion of Lactobacillus strain reduces anxiety and improves cognitive function in the hyperammonemia rat. Science China Life Sciences, 57 (3), 327-335. doi:10.1007/ s11427-014-4615-4

MacFabe, D. F., Cain, D. P., Rodriguez-Capote, K., Franklin, A. E., Hoffman, J. E., Boon, F., \& Ossenkopp, K. P. (2007). Neurobiological effects of intraventricular propionic acid in rats: Possible role of short chain fatty acids on the pathogenesis and characteristics of autism spectrum disorders. Behavioral Brain Research, 176 (1), 149-69.

Maes, M. (1999). Major depression and activation of the inflammatory response system. Advances in Experimental Medicine and Biology, 461, 25-46. 
Maes, M., Kubera, M., Leunis, J. C., \& Berk, M. (2012) Increased IgA and IgM responses against gut commensals in chronic depression: further evidence for increased bacterial translocation or leaky gut. Journal of Affective disorders, 141, 55-62. doi:10.1016/j.jad.2012.02.023

Martinowich, K., Manji, H., \& Lu, B. (2007). New insights into BDNF function in depression and anxiety. Nature Neuroscience, 10, 1089-93. doi:10.1038/ nn1971

Mayer, E. A., Knight, R., Mazmanian, S. K., Cryan, J. F., \& Tillisch, K. (2014). Gut microbes and the brain: Paradigm shift in Neuroscience. Journal of Neuroscience, 34(46): 15490-15696. doi:10.1523/JNEUROSCI.3299-14.2014

Myint, A. M., Kim, Y. K., Vertek, R., Scharpé, S., Steinbusch, H., \& Leonard, B. (2007). Kynurenine pathway in major depression: evidence of impaired neuroprotection. Journal of Affective Disorders, 98, 143-151. doi:1016/j. jad.2006.07.013

Ohland, C. L., Kish, L., Bell, H., Thiesen, A., Hotte, N., Pankiv, E., \& Madsen, K. L. (2013). Effects of Lactobacillus helveticus on murine behavior are dependent on diet and genotype and correlate with alterations in the gut microbiome. Psychoneuroendocrinology, 38, 1738 -1747. doi:10.1016/j. psyneuen.2013.02.008

Palomino, A., Vallejo-Illarramendi, A., Gonzalez-Pinto, A., Aldama, A., Gonzalez-Gomez, C., Mosquera, F., Gonzalez-Garcia, G., \& Matute, C. (2006). Decreased levels of plasma BDNF in first-episode schizophrenia and bipolar disorder patients. Schizophrenia Research, 86, 321-2. doi:10.1016/j. schres.2006.05.028

Park, H., Li, Z., Yang, X. O., Chang, S. H., Nurieva, R., Wang, Y. H., Hood, L., Tian, Q., \& Dong, C. (2005). A distinct lineage of CD4 T cells regulates tissue inflammation by producing interleukin 17. Nature Immunology, 6(11), 133-4.

Pessi, T., Isolauri, E., Sutas, Y., Kankaanranta, H., Moilanen, E., \& Hurme, M. (2001) Suppression of T-cell activation by Lactobacillus rhamnosus GG-degraded bovine casein. International Immunological pharmacology, 211-218. doi:10.1016/S1567-5769(00)00018-7

Quinn, T. C., Goodell, S. E., Fennell, C., Wang, S., Schuffler, M. D, Holmes, K. K., et al. (1984). Infections with Campylobacter jejuni and Campylobacter-like Organisms in Homosexual Men. Annals of Internal Medicine, 101,187-192. doi:10.7326/0003-4819-101-2-187

Rong, J., Zheng, H., Liu, M., Hu, X.,Wang, T., Zhang, X., Jin, F., \& Wang, L. (2015). Probiotic and anti-inflammatory attributes of an isolate Lactobacillus helveticus NS8 from Mongolian fermented koumiss. BMC Microbiology, 15, 196. doi:10.1186/s12866-015-0525-2

Rook, A. W. G., \& Lowry, A. W. (2008). The hygiene hypothesis and psychiatric disorders. Trends in Immunology. 29 (4), 150-158. doi:10.1016/j. it.2008.01.002

Shors, T., \& Matzel, D. (1997). Long-term potentiation: What's learning got to do with it? Behavioral and Brain Sciences, 20, 597-655.

Smith, K. S., \& Uwe, R. (2012). Review Anxiety and depression: Mouse genetics and pharmacological approaches to the role of GABAA receptor subtypes. Neuropharmacology, 62(1), 54-62. doi:10.1016/j.neuropharm.2011.07.026

Stanton, P. K., \& Sarvey, J. M. (1985). Depletion of Norepinephrine, But Not Serotonin, Reduces Long-term Potentiation in the Dentate Gyrus of Rat Hippocampal Slices. Journal of Neuroscience, 5(8), 2169-2176.

Steenbergen, L., Sellaro, R., Hemert, V. S., Bosch, A. J., \& Colzato, S. L. (2015). A randomized controlled trial to test the effect of multispecies probiotics on cognitive reactivity to sad mood. Brain, Behavior, and Immunity, 48, 258 264. doi:10.1016/j.bbi.2015.04.003.

Tsigos, C., \& Chrousos, G. P. (2002). Hypothalamic-pituitary-adrenal axis, neuroendocrine factors and stress. Journal of Psychosomatic Research, 53, 865871. doi:10.1016/S0022-3999(02)00429-4

Turner, J. R. (2009). Intestinal mucosal barrier function in health and disease. Nature Reviews Immunology, 9, 799-809. doi:10.1038/nri2653.

US Burden of Disease Collaborators. (2013). The state of US health, 1990-2010: burden of diseases, injuries, and risk factors. Journal of the American Medical Association, 310(6), 591-608.

Valladares, R., Bojilova, L., Potts, A. H., Cameron, E., Gardner, C., Lorca, G.,
\& Gonzalez, C. F. (2013). Lactobacillus johnsonii inhibits indoleamine 2,3-dioxygenase and alters tryptophan metabolite levels in BioBreeding rats. Federation of American Societies for Experimental Biology; 27(4), 1711-20. doi:10.1096/fj.12-223339

Valladares, R., Sankar, D., Li, N., Williams, E., Lai, K. K., Abdelgeliel, A. S., Gonzalez, C. F... \& Lorca, G. L. (2010). Lactobacillus johnsonii N6.2 Mitigates the Development of Type 1 Diabetes in BB-DP Rats. PLoS ONE; 5(5): e1057. doi:10.1371/journal.pone.0010507

Vijayakumar, M., \& Meti, B. L. (1999). Alterations in the levels of monoamines in discrete brain regions of clomipramine-induced animal model of endogenous depression. Neurochemical Research, 24 (3), 345-349. doi:10.1023/A:102099231 